\title{
Structural and Functional Determinants of AC8 Trafficking, Targeting and Responsiveness in Lipid Raft Microdomains
}

\author{
Valentina G. Tabbasum ${ }^{1}$ - Dermot M. F. Cooper ${ }^{1}$ D
}

Received: 14 September 2018 / Accepted: 21 January 2019 / Published online: 12 February 2019

(c) The Author(s) 2019

\begin{abstract}
The fidelity of cAMP in controlling numerous cellular functions rests crucially on the precise organization of cAMP microdomains that are sustained by the scaffolding properties of adenylyl cyclase. Earlier studies suggested that AC8 enriches in lipid rafts where it interacts with cytoskeletal elements. However, these are not stable structures and little is known about the dynamics of AC8 secretion and its interactions. The present study addresses the role of the cytoskeleton in maintaining the AC8 microenvironment, particularly in the context of the trafficking route of AC8 and its interaction with caveolin1. Here, biochemical and live-cell imaging approaches expose a complex, dynamic interaction between AC8 and caveolin1 that affects AC8 processing, targeting and responsiveness in plasma membrane lipid rafts. Site-directed mutagenesis and pharmacological approaches reveal that AC8 is processed with complex $\mathrm{N}$-glycans and associates with lipid rafts en route to the plasma membrane. A dynamic picture emerges of the trafficking and interactions of AC8 while travelling to the plasma membrane, which are key to the organization of the AC8 microdomain.
\end{abstract}

Keywords Adenylyl cyclase $\cdot$ cAMP $\cdot$ Caveolin $\cdot$ Lipid rafts $\cdot$ Plasma membrane $\cdot$ Cholesterol

\section{Introduction}

The residence of adenylyl cyclases (ACs) at the plasma membrane is a central premise of cAMP signalling, although their presence there is much understudied. The ever-expanding complexity of intracellular protein trafficking pathways is being elucidated from growing insights on biosynthesis, post-translational processing and molecular assembly of membrane proteins and their vesicular trafficking and endocytosis (Bonifacino and Glick 2004; Doherty and McMahon 2009; Vagin et al. 2009; Parton and Howes 2010; Feige and Hendershot 2013). However, little is known about trafficking routes of ACs and their targeting and compartmentalization to specific plasma membrane microdomains (Gu et al. 2001,

Electronic supplementary material The online version of this article (https://doi.org/10.1007/s00232-019-00060-x) contains supplementary material, which is available to authorized users.

Dermot M. F. Cooper

Dmfc2@cam.ac.uk; gianatabbasum@hotmail.com

1 Department of Pharmacology, University of Cambridge, Tennis Court Rd., Cambridge CB2 1PD, UK
2002; Swaney et al. 2006; Cooper and Tabbasum 2014, Johnstone et al. 2018).

Nine membrane-bound (1-9) and a soluble AC (10) have been cloned and characterized (Cooper 2003). Among these species, adenylyl cyclase 8 (AC8) which is confined to neuronal and secretory tissue has been implicated in long-term memory and long-term potentiation (Cali et al. 1994; Ferguson and Storm 2004; Conti et al. 2007). AC8, which is activated by calcium $\left(\mathrm{Ca}^{2+}\right)$ acting via calmodulin $(\mathrm{CaM})$, in intact cells displays a specific dependence on $\mathrm{Ca}^{2+}$ entering the cell through store-operated $\mathrm{Ca}^{2+}$-entry (SOCE) processes in non-excitable cells (Fagan et al. 1996). The SOCE process involves translocation of the endoplasmic reticulum (ER) $\mathrm{Ca}^{2+}$ sensor, stromal interaction molecule 1 (STIM1) upon depletion of $\mathrm{Ca}^{2+}$ stores to the plasma membrane, where it binds to and activates the channel component, Orai1, which permits an inward extracellular $\mathrm{Ca}^{2+}$ (SOCE) current (Parekh and Putney 2005) that activates AC8. The strict dependence of AC8 on regulation by SOCE relies on direct binding of its $\mathrm{N}$-terminus (AC8-Nt) to the $\mathrm{N}$-terminus of Orai1, which resides, along with AC8, in the so-called lipid raft domains of the plasma membrane (Willoughby et al. 2012). 
In their natural state, biological membranes are dynamically heterogeneous, both in terms of their chemical composition and physical properties. Lipid rafts are envisaged as dynamic assemblies of cholesterol and sphingolipids in the plane of the membrane (Brown and London 1998; Simons and Vaz 2004; Sezgin et al. 2017). The lipid and protein composition of rafts has consequences for their biophysical as well as biochemical properties that differ from nonraft domains. Lipid rafts are difficult to detect in live cells except with the use of labelled probes by elegant biophysical methods and modern microscopic techniques. Detailed exploration of the nature of rafts and their role in trafficking signalling molecules-particularly through a range of cellular compartments where they undergo covalent modifications-is not readily accessible (Lagerholm et al. 2005; Levental and Veatch 2016; Sezgin et al. 2017). In order to address these issues, a combination of biochemical and livecell methods combined with rigorous statistical assessments of the consequences of pharmacological manipulations and for the responsiveness of the adenylyl cyclase to physiological regulation must be adopted. That strategy is adopted in the current investigation. The fluctuating nature and nanometer scale of lipid rafts, taxes the isolation of uncontaminated lipid raft microdomains for proteomics analysis (Mohamed et al. 2018). Nevertheless, in the last decade, a considerable number of studies using an array of biochemical extraction methods in reporting the proteomic analysis of bulk lipid rafts prepared from diverse cells and tissues have arrived at a reasonable consensus of raft-resident proteins [A searchable database for mammalian lipid raft proteomics data (RaftProt) has been developed (Shah et al. 2015). Of note, within the present context, both AC8 and Orai1 are encountered in that database.].

Caveolae are transient platforms that scaffold and compartmentalize characteristic proteins to initiate diverse molecular signalling routes (Cohen et al. 2004). The scaffolding properties of caveolins residing in caveolae are generally mediated by the so-called caveolin scaffolding domain (CSD). This 20 amino-acid stretch located at the Nt of caveolins binds to and often suppresses the downstream signalling of caveolin-interacting molecules (Razani et al. 2002).

Soluble, intracellular pools of caveolin have been identified in various subcellular compartments, which suggests a role of caveolae in intracellular trafficking (Kurzchalia et al. 1992; Dupree et al. 1993; Gagescu et al. 2000; Pol et al. 2005). Indeed, caveolae permit intracellular trafficking of intra- and extracellular elements through biosynthetic, transcytotic and endocytotic routes (Bastiani and Parton 2010).

The sophisticated organization of AC8 and its partitioning alongside components of cAMP microdomains in lipid rafts is required for its regulation by SOCE. Perturbing the integrity of lipid rafts alters both the basal and stimulated activity of $\mathrm{AC} 8$, along with its mobility in the plasma membrane, which underscores the importance of these domains in AC8 function and distribution (Smith et al. 2002; Ayling et al. 2012). Various determinants, such as N-linked glycosylation, cholesterol recognition amino-acid sequence (CRAC) motifs, binding to the cytoskeleton and tethering by un-identified scaffolds, have been envisaged to localize AC8 to these domains (Pagano et al. 2009, Ayling et al. 2012). At the same time, limited information exists regarding the biosynthesis, post-translational processing and trafficking routes of AC8 (Gu et al. 2001; Crossthwaite et al. 2005; Cooper and Crossthwaite 2006).

Consequently, in the present study, we investigate the mechanisms which traffic, target and segregate AC8 to lipid rafts, alongside the ramifications of such events on AC8 responsiveness. A potential association of AC8 with caveolin 1 is investigated, as well as re-addressing the role of N-linked glycosylation of AC8 and its associations with the actin cytoskeleton, as a possible means by which AC8 is recruited to lipid rafts. By using biochemical, high-resolution microscopy, mutagenesis and pharmacological tools, we suggest that AC8 assembly into lipid raft microdomains is a multi-step process that relies on numerous structural and functional elements, whose complexity has not hitherto been appreciated.

\section{Experimental Procedures}

\section{Constructs}

Epac2-camps was a gift from Martin Lohse (Würzberg University, Germany) (Nikolaev et al. 2004). AC8-HA was created by Dr. Antonio Ciruela by PCR, and this insert was cloned into pcDNA 3.0 between the sites Kpn1 and Xba1. YFP-AC8 was generated by cloning AC8 cDNA between the Apa1 and Xba1 restriction sites of pEYFP-C1. GFPAC8 ${ }^{\mathrm{N} 814 \mathrm{Q}, \mathrm{N} 818 \mathrm{Q}, \mathrm{N} 855 \mathrm{E}}$ (GFP-AC8 $\Delta \mathrm{N}$-gly) was generated by Pagano et al. (2009).

YFP-AC8 Y144A, F151A, F1144A, Y1146A, Y1151A (YFP-AC $8 \Delta$ cav) was generated by Dr. Jessica Sorge by sitedirected mutagenesis according to the QuickChange protocol (Stratagene, La Jolla, CA) using the fusion high-fidelity polymerase kit (Finnzymes) according to the manufacturer's instructions using YFP-AC8 as a template. $\mathrm{AC} 8^{\mathrm{Y} 144 \mathrm{~A}}$ was introduced using the forward and reverse primer sequence, 5'-CTCGGATTTCTTCCTCAATGGGGGAGCCAGCGC CCGTGGGGTCATTT- ${ }^{\prime}$ ' and 5'-AAATGACCCCACGGG CGCTGGCTCCCCCATTGAGGAAGAAATCCGAG - 3' ,respectively. $\mathrm{AC} 8^{\mathrm{F} 151 \mathrm{~A}}$ was introduced using the forward primer sequence, 5'-CAGCGCCCGTGGGGTCATTGCCCC AACCCTA- ${ }^{\prime}$ ' and reverse, 5'-TAGGGTTGGGGCAATGAC CCCACGGGCGCTG-3'. AC8 ${ }^{\mathrm{F} 1144 \mathrm{~A}}$ was introduced using the forward and reverse primer sequence, $5^{\prime}$-GATCAGGGC 
TTTGCCGCCGACTACCGGGGAGA-3' and 5'-TCTCCC CGGTAGTCGGCGGCAAAGCCCTGATC- $3^{\prime}$. AC8 ${ }^{\mathrm{Y} 1146 \mathrm{~A}}$ was introduced using the forward and reverse primer sequence, 5'- AGGGCTTTGCCGCCGACGCCCGGGGAG AGATATATG-3' and 5'-CATATATCTCTCCCCGGGCGT CGGCGGCAAAGCCCT-3'. AC $8^{\mathrm{Y} 1151 \mathrm{~A}}$ was introduced using the forward and reverse primer sequence, $5^{\prime}$-CCGCCG ACGCCCGGGGAGAGATAGCTGTGAAGGGCAT-3' and 5'-ATGCCCTTCACAGCTATCTCTCCCCGGGCGTCG GCGG-3'.

\section{Cell Culture and Transfections}

Stable expression of constructs was achieved as previously described (Delint-Ramirez et al. 2015). For single-cell cAMP measurements, HEK293 cells (European Collection of Cell Culture) were transfected with $0.5 \mu \mathrm{g}$ of YFP-AC8, YFP-AC $8 \Delta$ cav or GFP-AC8 $\Delta \mathrm{N}$-gly and $1 \mu \mathrm{g}$ of Epac2camps sensor using the Lipofectamine 2000 (Invitrogen) transfection method according to the manufacturer's instructions. For knock-downs, either caveolin1 small interfering (si) RNA or control siRNA (Santa Cruz Biotechnology) was added at $30 \mathrm{nM}$ final concentration following the manufacturer's instructions. Real-time measurements of cAMP were performed 48-h post-transfection.

\section{Single-Cell FRET Measurements}

Real-time FRET measurements were performed using an EM CCD Ixon, an EMCCD camera (Andor, Belfast) and an Optosplit (505DC) (Cairn Research) as previously described (Willoughby et al. 2010). The Optosplit divides the CFP $(470 \mathrm{~nm})$ and YFP $(535 \mathrm{~nm})$ emission images. Cells were excited at $435 \mathrm{~nm}$ using a monochromator (Cairn Research) and 51,017 filter set (Chroma Technology Corp) attached to a Nikon eclipse TE2000-S microscope $(\times 40$ oil-immersion objective) for dual-emission ratio imaging. Image processing was performed as described previously (Willoughby et al. 2012). Single-cell FRET data were plotted as changes in background subtracted $470 \mathrm{~nm}$ versus $535 \mathrm{~nm}(C F P / Y F P)$ emission ratio relative to maximum FRET ratio change recorded with saturating cAMP concentrations (maximum; Max) concentration attained by addition of a cocktail of $10 \mu \mathrm{M}$ Forskolin and $100 \mu \mathrm{M}$ IBMX.

\section{Lipid Raft Isolation}

Cold detergent extraction of lipid rafts and flotation on sucrose density was carried out as previously described (Delint-Ramirez et al. 2015).

\section{Immunoblotting}

Proteins were resolved using 6, 10 or $12 \%$ SDS-polyacrylamide gels as previously described (Delint-Ramirez et al. 2015; Willoughby et al. 2012). Following blocking, membranes were incubated overnight at $4{ }^{\circ} \mathrm{C}$ with the following antibodies: anti-caveolin antibody (1:2000, BD Biosciences), anti-GFP antibody (1:10,000, Abcam), anti$\beta$-adaptin antibody (1:5000, Santa Cruz Biotechnology), anti-actin antibody (1:1000, Sigma) and anti-HA (1:5000, Sigma) or anti-GST antibody (1:40,000; Sigma) in TTBS containing $1 \%(w / v)$ skimmed milk.

The membranes were incubated for $1 \mathrm{~h}$ with the following secondary antibodies: goat anti-mouse IgG conjugated to horseradish peroxidase $(1: 20,000$, Promega for anti-HA, anti-GST, anti-GFP and anti-actin) or goat anti-rabbit IgG conjugated to horseradish peroxidase (1:20,000, Fisher Scientific, for anti-caveolin and anti- $\beta$-adaptin) in TTBS containing 5\% $(w / v)$ skimmed milk. Detection of proteins and quantification of immunoreactive bands were performed as previously described (Delint-Ramirez et al. 2015).

\section{Cell Treatments and Immunocytochemistry}

Cells were plated onto 22-mm poly-L-lysine-coated coverslips and treated with the indicated drugs after $24 \mathrm{~h}$. Where appropriate, cells were incubated with $2 \mathrm{mM}$ Latrunculin B (Lat B; Merck), $10 \mathrm{mM}$ methyl- $\beta$-cyclodextrin (M $\beta C D$; Sigma) or $5 \mu \mathrm{g} / \mathrm{ml}$ Brefeldin A (BFA; Sigma) for $1 \mathrm{~h}$ at $37^{\circ} \mathrm{C}$. Cells were fixed and permeabilized as previously described (Ayling et al. 2012).

For the detection of endogenous caveolin1, cells were incubated with PBS containing $0.5 \%$ goat serum; and anticaveolin antibody (1:1000, BD Biosciences) for $1 \mathrm{~h}$ at $37^{\circ} \mathrm{C}$. Coverslips were rinsed three times with PBS for $5 \mathrm{~min}$ and incubated with PBS containing $0.5 \%$ goat serum and AlexaFluor555-conjugated goat anti-rabbit antibody (1:200, Invitrogen) for $1 \mathrm{~h}$ at $37^{\circ} \mathrm{C}$. Coverslips were rinsed and mounted using Hardest Vectashield with DAPI (Vector Laboratories) and stored at $4{ }^{\circ} \mathrm{C}$. Staining of actin filaments was achieved by blocking the coverslips with PBS containing $1 \%$ BSA for 30 min at $37^{\circ} \mathrm{C}$ and then incubation in PBS containing BSA; $1 \%$ and AlexaFluor568-conjugated phalloidin $\left(1 \mathrm{U} / \mathrm{ml}\right.$, Invitrogen) for $2 \mathrm{~h}$ at $37^{\circ} \mathrm{C}$. Staining with phalloidin was performed on fixed, permeabilized cells. Visualization of plasma membranes stained with CellMask ${ }^{\mathrm{TM}}$ Deep Red (Invitrogen) was performed in live cells. HEK293 cells were incubated with media containing CellMask ${ }^{\mathrm{TM}}$ Deep Red ( $5 \mu \mathrm{g} / \mu \mathrm{l}$, Invitrogen) for $10 \mathrm{~min}$ at $37{ }^{\circ} \mathrm{C}$. For staining with WGA, cells were incubated with AlexaFluor555conjugated WGA ( $5 \mu \mathrm{g} / \mathrm{ml}$; Invitrogen) for $10 \mathrm{~min}$ at $37^{\circ} \mathrm{C}$. Cells were then washed and mounted as described above. 


\section{Confocal Imaging}

Confocal images were captured with a Leica SP5 TCS laser scanning confocal microscope attached to a DM16000 inverted microscope, equipped with a $\times 63$ plan-apochromatic 1.4 NA oil-immersion objective (Leica Microsystems) using the LAS AF Leica software version 1.8.2. All images were captured at 1024 resolution and $100 \mathrm{~Hz}$. Optical slice thicknesses of either $1.074 \mu \mathrm{m}$ or $0.733 \mu \mathrm{m}$ are indicated in the figure legends.

The GFP and YFP-tagged constructs were imaged using the $488 \mathrm{~nm}$ and the $514 \mathrm{~nm}$ excitation bands of an Argon laser. Images were collected at 493-550 nm or 520-600 nm emission bandwidths, respectively. For visualization of the cell nuclei, DAPI was excited with the UV $405 \mathrm{~nm}$ laser and images were collected using 410-480 $\mathrm{nm}$ emission spectra. Visualization of plasma membranes stained with CellMask ${ }^{\mathrm{TM}}$ Deep Red was obtained by exciting the dye with the HeNe633 laser (emission spectra 640-700 nm). The endogenous caveolin1 stained with AlexaFluor555-conjugated goat anti-rabbit antibody, the actin filaments stained with AlexaFluor568-conjugated phalloidin and the structures stained with AlexaFluor555-conjugated WGA were visualized using the HeNe543 laser with an emission bandwidth between 573 and $630 \mathrm{~nm}$.

\section{Image and Statistical Analysis}

ImageJ analysis to determine colocalization coefficient $(R r)$ was performed as previously described (Ayling et al. 2012). Pearson's coefficient ranges from -1 to 1 , where -1 represents perfect non-colocalization, 0 means that the signal is entirely random and 1 suggests that the images colocalize perfectly. The signal from each fluorophore was collected sequentially with appropriate controls in order to eliminate bleed-through; the images captured are not saturated. For analysis, the selection was outlined using a polygon selection and the outside cleared for both images to ensure that only the cell of interest is analysed.

Statistical significance of colocalization $(R r)$, densitometry (arbitrary units; AU) and real-time FRET measurements were determined by GraphPad Prism software using Student's $t$ tests with Welch's correction. In each case, the number in brackets $(n)$ refers to the number of cells measured in at least three separate experiments, or the number of times the experiment was repeated, as appropriate. Data are presented as the mean \pm standard error of the mean (SEM) or mean $\pm \mathrm{SE}$ (as indicated) with significance set at $p<0.05$, where $* p<0.05$; $* * p<0.01$; $* * * p<0.001, * * * * p<0.0001$.

\section{Results}

\section{Colocalization of Caveolin1 and AC8 in Lipid Rafts Depends on the Cytoskeleton}

Previous studies showed that AC8 both co-sediments with caveolin1 on sucrose density gradients following cold detergent extraction (Smith et al. 2002) and also appears to bind to actin microfilaments at the plasma membrane (Ayling et al. 2012). Thus, given the known interaction between caveolin and the cytoskeleton (Muriel et al. 2011), we hypothesized that cytoskeletal disruption might affect the colocalization of AC8 and caveolin1.

HEK293 cells stably expressing YFP-tagged AC8 were incubated with Lat B, a macrolide that binds to G-actin and causes passive cytoskeletal depolymerization by sequestering actin monomers and inhibiting ATP/ADP exchange on actin (Spector et al. 1989; Yarmola et al. 2000). Following the treatment, cells were lysed with $1 \%$ Triton X-100 and lipid raft fractions were isolated from the soluble extract following sucrose density ultracentrifugation. The protein-todetergent ratio used in this procedure allows the detection of subtle changes in the lipid raft fraction, while changes in the soluble fractions are unlikely to be detected (Delint-Ramirez et al. 2015). Five equal-volume fractions and the pellet were collected and analysed side-by-side with the soluble extract by immunoblotting (Fig. 1a). As expected, AC8 and caveolin1 were enriched in the lipid rafts fraction. Remarkably, a pool of actin also concentrated in these rafts. Actin, caveolin 1 and AC8 were also detected in the soluble extract and the pellet (Fig. 1a). Upon dissolving the cytoskeleton with Lat B, the levels of actin in the lipid raft fractions were significantly reduced. Under these conditions, the levels of AC8 and caveolin1 were also decreased in lipid rafts. The levels of proteins analysed in the soluble extract or pellet were not detectably altered by these treatments (Fig. 1a, b).

Previous reports suggested that AC8 remains associated with actin despite cytoskeletal disruption (Ayling et al. 2012). In the present study, imaging experiments confirmed that in intact cells, AC8 along with F-actin stained with phalloidin colocalized at the plasma membrane (Fig. 1c). To resolve the degree of colocalization, Pearson's coefficient $(R r)$ was determined as described previously (Ayling et al. 2012). Since our study is assessing a functional relationship between two proteins, Pearson's coefficient is the most appropriate metric to do so. Pearson's is in fact superior to other colocalization metrics (Adler and Parmryd 2010) and is currently one of the most extensively used metric to determine colocalization on confocal images (Pike et al. 2017, Aaron et al. 2018). At rest, AC8 displayed a high degree of colocalization with the phalloidin (F-actin) signal $(R r=0.75$, Fig. 1c, d). The endogenous caveolin1 detected with a specific antibody presented as two 

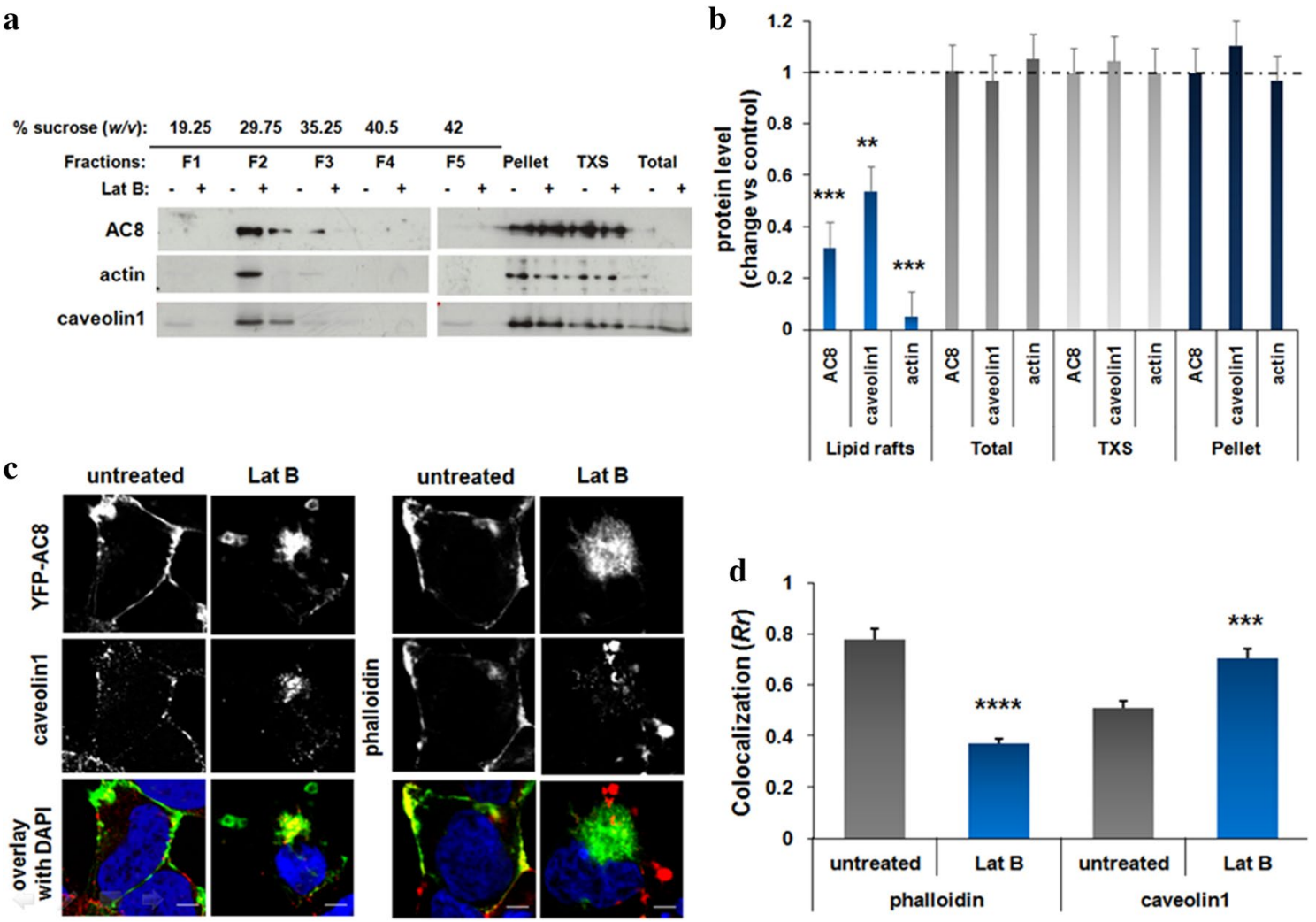

Fig. 1 Dissolving the actin cytoskeleton modulates the colocalization between AC8 and caveolin1 a HEK293 cells stably expressing YFP-AC8 were homogenized in lysis buffer with $1 \%$ Triton X-100. The Triton X-100 soluble (TXS) and insoluble fractions were isolated, and $20 \mu \mathrm{g}$ of the extracts was analysed by Western blotting. b Densitometric analysis (AU) of blots expressed as the ratio of protein after Lat B $\left(2 \mu \mathrm{M}, 1 \mathrm{~h}, 37^{\circ} \mathrm{C}\right)$ treatment over control (DMSO).

distinct pools, one at the plasma membrane and one intracellular, forming micro-aggregates (Fig. 1c); as had also been observed by Pol et al. (2005). As earlier observed by Ayling et al. (2012), cytoskeletal disruption caused the actin microfilaments to collapse and AC8 aggregated into a cytosolic region proximal to the cell nucleus showing less colocalization with the actin microfilaments than in intact cells $(R r=0.37$; Fig. 1c, d). When the distribution of caveolin1 was examined under these conditions, it was seen that caveolin1 internalized and colocalized with AC8 to a greater extent $(R r=0.70)$ than in resting cells $(R r=0.51 ;$ Fig. $1 \mathrm{c}, \mathrm{d})$. Thus, these data suggest that cytoskeletal re-arrangements modulate the co-distribution of AC8 with caveolin1 and their recruitment to lipid rafts.

\section{Knock-Down of Caveolin1 Alters the Behaviour of AC8 on Sucrose Gradients and Its Responsiveness to SOCE}

The functional interaction between AC8 and caveolin 1 was explored by knock-down of caveolin1 using siRNA.
Data are presented as mean $\pm \mathrm{SE}(n=3)$. c Representative confocal imaging of HEK293 cells expressing YFP-AC8 treated or not with Lat $\mathrm{B}$ and stained with phalloidin $(n=18-20)$ or caveolin1 antibody $(n=13-15$; scale bars represent $10 \mu \mathrm{m}$; optical section thickness $=1.073 \mu \mathrm{m})$. $\mathbf{d}$ Colocalization coefficient $(R r)$ of $\mathbf{c}$. Data are presented as mean \pm SEM

HEK293 cells stably expressing YFP-AC8 were incubated with either caveolin 1 siRNA or control (scrambled) siRNA for $24 \mathrm{~h}$. Densitometric analysis determined that caveolin1selective siRNA-reduced caveolin1 levels in HEK293 cells by approximately $40 \%$ compared to control siRNA. The levels of expression of AC8 and actin were not affected by the caveolin1 siRNA knock-down (Fig. 1Sa).

Caveolin1 is regarded as a general kinase inhibitor (Razani et al. 2002). And previous reports suggest that caveolins negatively regulate cAMP signalling (Allen et al. 2009; Sato et al. 2012). To determine a possible functional impact of caveolin1 knock-down on AC8 activity, realtime measurements of cAMP using the Förster resonance energy transfer (FRET)-based cAMP sensor Epac2-camps were performed following caveolin1 siRNA interference. To exploit the strict dependence of AC8 on SOCE channels, SOCE was triggered pharmacologically via passive irreversible depletion of $\mathrm{Ca}^{2+}$ stores by addition of thapsigargin ( $T_{\mathrm{g}}$ - a sarcoplasmic/endoplasmic reticulum $\mathrm{Ca}^{2+}$ ATPase inhibitor) followed by addition of $\left[\mathrm{Ca}^{2+}\right]_{\mathrm{ex}}$ as previously 
described (Ayling et al. 2012). Cells transfected with control siRNA displayed a significant cAMP response to SOCE; this response was significantly increased in cells that had been transfected with caveolin1 siRNA (Fig. 1Sb, c). Thus, it appears that caveolin 1 exerts a negative influence on AC8 activity.

Next, AC8 distribution following caveolin1 knock-down was assessed by cold detergent extraction as described above. Knock-down of caveolin 1 affected the integrity of lipid rafts. Under these conditions, a subtle decrease in AC8 immunoreactivity in the lipid rafts fractions was observed which was mirrored by actin and caveolin 1 immunoreactivity (Fig. 2a, b). In parallel, imaging data revealed that neither AC8 nor actin changed distribution in cells in which caveolin1 was knocked down. However, a significant decrease in AC8 colocalization with caveolin 1 was detected $(R r=0.30$; Fig. 2c, d). Taken together, these data can be taken to indicate that caveolin1 maintains the integrity of the AC8 microenvironment and can act as a negative regulator of SOCEmediated AC8 activity.

\section{Mutations in the Caveolin-Binding Sites Impair AC8 N-Linked Glycosylation, Its Plasma Membrane Targeting and Functionality}

The persistent functional impact of caveolin1 knock-down on AC8 organization and responsiveness prompted us to search for and investigate residues in the AC8 sequence that might mediate caveolar recruitment. Many proteins that reside in caveolae display 'caveolin-binding motifs' (CBM) which are 8-11 hydrophobic polyaromatic stretches with loose, yet identifiable sequences. These sequences are viewed to mediate caveolar recruitment through direct interactions with the CSD (Couet et al. 1997).

Two putative CBM were identified in AC8. The CBMs located at the Nt (residues 144-151) displayed the sequence, YSYRGVIF and at the Ct (residues 1144-1151) the sequence, FDYRGEIY. These CBMs conform to the fol-

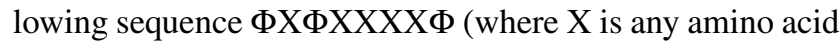
and $\Phi$ is an aromatic residue). Within the CBM, it is the aromatic residues that have been demonstrated to mediate

a
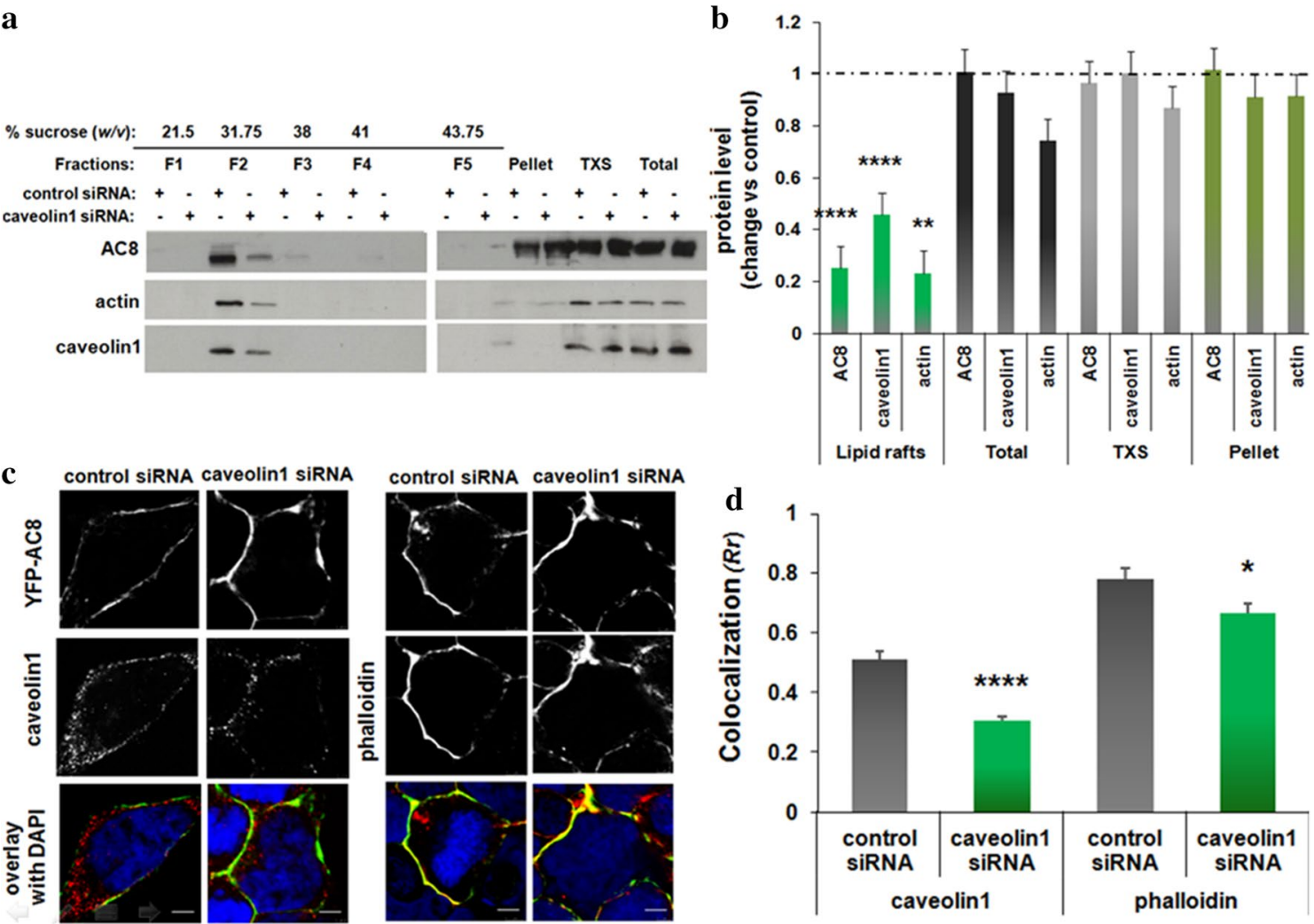

Fig. 2 Knock-down of caveolin1 alters AC8 distribution a HEK293 cells stably expressing YFP-AC8 were homogenized in lysis buffer with $1 \%$ Triton $\mathrm{X}-100$ following transfection of either caveolin1 siRNA $(30 \mathrm{nM})$ or control siRNA $(30 \mathrm{nM})$ for $24 \mathrm{~h}$. The Triton X-100 soluble (TXS) and insoluble fractions were isolated, and $20 \mu \mathrm{g}$ of the extracts was analysed by SDS-PAGE. b Densitometric analysis (AU) of blots expressed as the ratio of protein after knock-down of caveo- lin1 over control. Data are presented as mean \pm SE $(n=3)$. c Confocal imaging of HEK293 cells expressing YFP-AC8 transfected with either caveolin1 siRNA or control siRNA and stained with phalloidin $(n=25-30)$ or caveolin1 antibody $(n=19-24$; scale bars represent $10 \mu \mathrm{m}$; optical section thickness $=1.073 \mu \mathrm{m})$. d Colocalization coefficient $(R r)$ of $\mathbf{c}$. Data are presented as mean \pm SEM 
the interaction with caveolin proteins (Couet et al. 1997). By site-directed mutagenesis, point mutations in the aromatic residues in the CBM motifs of AC8 were introduced to generate $\mathrm{AC} 8^{\mathrm{Y} 144 \mathrm{~A}, \mathrm{~F} 151 \mathrm{~A}, \mathrm{~F} 1144 \mathrm{~A}, \mathrm{Y} 1146 \mathrm{~A}, \mathrm{Y} 1151 \mathrm{~A} \text {; we shall refer to }}$ this construct as YFP-AC $8 \Delta$ cav hereafter (Fig. 3a).

In order to determine how YFP-AC8 $\Delta$ cav was expressed, the construct was transfected into HEK293 cells and lysates were analysed by immunoblotting. Over-expression of YFP-AC $8 \Delta$ cav yielded two immunoreactive bands, which represented the glycosylated (more diffuse band) and the non-glycosylated species (a sharper, lower molecular weight band; Fig. 3b). Indeed, native (transfected) AC8 migrates as two individual species with a more prominent diffuse band running at $165 \mathrm{kDa}$ and a lower molecular weight specie running at $125 \mathrm{kDa}$ corresponding to the glycosylated and

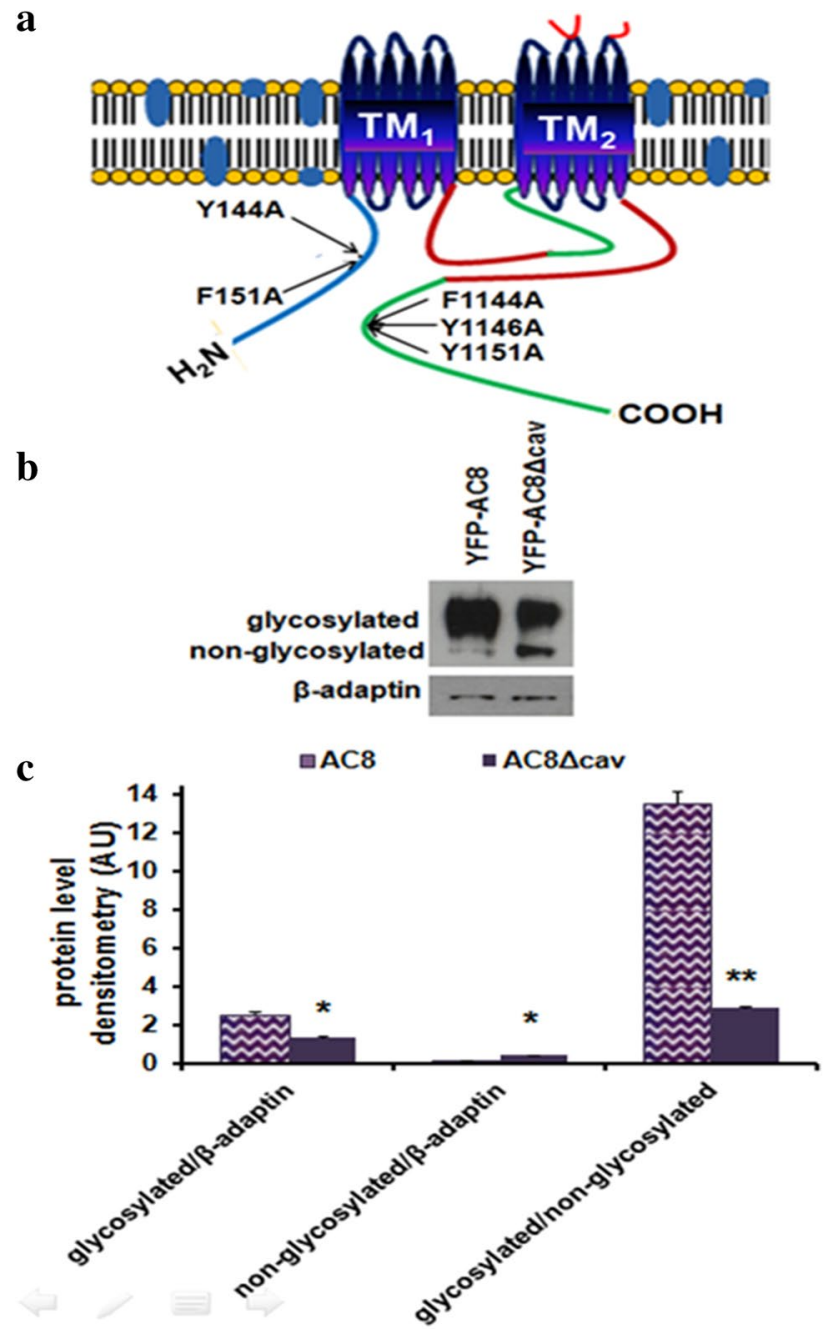

Fig. 3 Expression pattern of YFP-AC8 $\Delta$ cav a Schematic representation of YFP-AC8 $\Delta$ cav (mutations indicated by arrows). b Western blot analysis of crude membranes from HEK293 cells expressing YFP-AC $8 \Delta$ cav. c Densitometric analysis (AU) of $\mathbf{b}(n=4)$. Data are presented as mean \pm SEM non-glycosylated species respectively (Pagano et al. 2009). Compared with native AC8 in the case of YFP-AC $8 \Delta$ cav, the band representing the non-glycosylated species was a lot more prominent than the glycosylated species (Fig. 3b). Quantification of the immunoreactive bands corresponding to YFP-AC8 and YFP-AC8 $\Delta$ cav species by scanning densitometry determined that YFP-AC8 $\Delta$ cav was statistically less glycosylated compared to YFP-AC8 (Fig. 3c).

In order to determine whether these mutations affect the targeting of the AC8, HEK293 cells stably expressing YFPAC $8 \Delta$ cav were analysed by confocal microscopy. Live-cell imaging indicated that a pool of YFP-AC8 $\Delta$ cav did not overlay with the CellMask ${ }^{\mathrm{TM}}$ fluorescent signal, which suggested that at least partly, YFP-AC8 $\Delta$ cav was not confined to the plasma membrane. This observation was also confirmed by determining the colocalization coefficient $(R r=0.49$; Fig. 2Sa, b). These findings imply that the CBM may play an important role in the distribution of AC8 at the plasma membrane.

Given the dissimilarities in terms of the glycosylation profile between $\mathrm{AC} 8$ and $\mathrm{AC} 8 \Delta$ cav, it seemed appropriate to determine whether the mutations in the CBM perturb AC8 organization in lipid rafts. Therefore, lipid raft fractionation of HEK293 cells stably expressing YFP-AC8 $\Delta$ cav was performed before and after treatment with Lat B as previously described. Unexpectedly, in resting cells, YFP-AC $8 \Delta$ cav co-immunopurified along with caveolin1 on sucrose gradients (Fig. 4a). Moreover, just like in the case of native YFP-AC8 (Fig. 1), disrupting the actin microfilaments lowered the affinity of YFP-AC $8 \Delta$ cav for lipid rafts (Fig. 4a, b). Next, we assessed the impact of the CBM mutations on AC8 functionality by measuring the cAMP production following SOCE in HEK293 cells expressing AC8 $\Delta$ cav. Realtime measurements of cAMP using Epac2-camps revealed that by statistically robust criteria, AC $8 \Delta$ cav was approximately $40 \%$ less responsive to SOCE than full-length AC8. At the same time, a delay in the responsiveness of AC $8 \Delta \mathrm{cav}$ was recorded (Fig. 4c, d). Taken together, these data imply the CBM may not be involved in the recruitment of AC8 to caveolae. However, given that the mutations affected AC8 responsiveness to SOCE, our data suggest that other mechanisms, besides the necessity of residence in lipid rafts may be required for full AC8 functionality.

\section{$\mathrm{N}$-Linked Glycosylation Targets AC8 to the Plasma Membrane but not to Lipid Rafts}

Since AC $8 \Delta$ cav displayed altered $\mathrm{N}$-linked glycosylation profiles, targeting and activity, we next investigated the likely link between $\mathrm{N}$-linked glycosylation and AC8 functionality. For this purpose, we used a mutant of AC8, in which the three putative $\mathrm{N}$-glycosylation asparagine residues were mutated to generate GFP-AC8 ${ }^{\mathrm{N} 814 \mathrm{Q}, \mathrm{N} 818 \mathrm{Q}, \mathrm{N} 855 \mathrm{E}}$, 

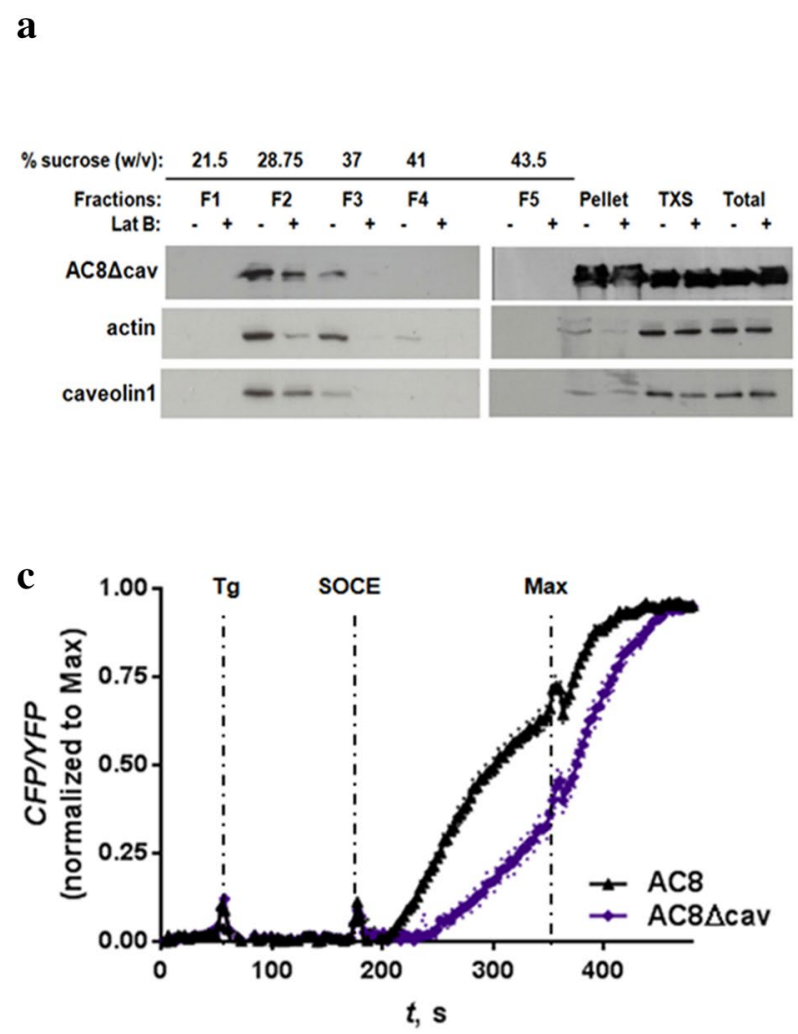

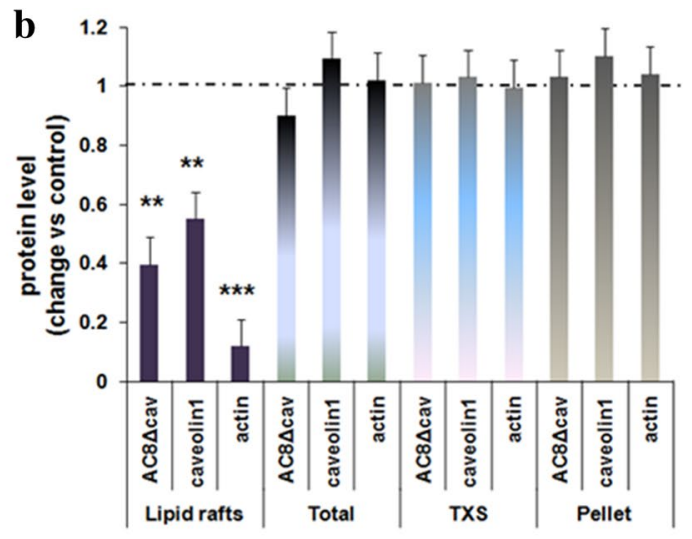

d

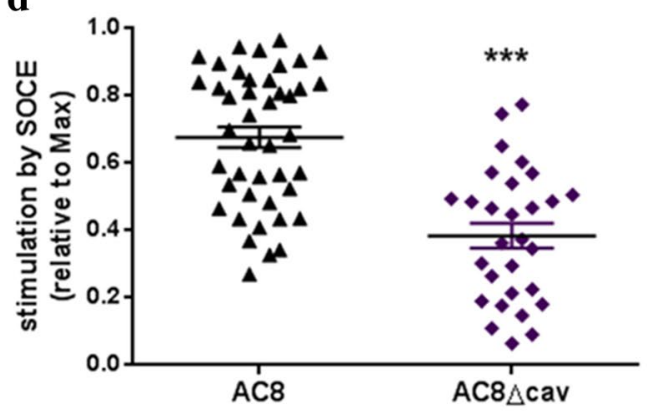

Fig. 4 Mutations in the CBM alter AC8 responsiveness a HEK293 cells stably expressing YFP-AC8 $\Delta$ cav were homogenized in lysis buffer with $1 \%$ Triton X-100. The Triton X-100 soluble (TXS) and insoluble fractions were isolated, and $20 \mu \mathrm{g}$ of the extracts was analysed by SDS-PAGE. b Densitometric analysis (AU) of blots expressed as the ratio of protein after Lat B $\left(2 \mu \mathrm{M}, 1 \mathrm{~h}, 37^{\circ} \mathrm{C}\right)$ treatment over control (DMSO). Data are presented as mean $\pm \mathrm{SE}(n=3)$.

described formerly by Pagano et al. (2009) and termed hereafter AC $8 \Delta \mathrm{N}$-gly, in which $\mathrm{N}$-linked glycosylation was precluded (Fig. 5a).

As previously determined, stable expression of AC $8 \Delta \mathrm{N}$-gly yielded a single immunoreactive band on Western blots, corresponding to the non-glycosylated AC8 (Fig. 5b). Stably expressed AC $8 \Delta \mathrm{N}$-gly largely, but not entirely, exhibited a plasma membrane-like distribution. Careful colocalization studies determined that the AC $8 \Delta \mathrm{N}$-gly fluorescent signal showed substantially less overlay with the plasma membrane fluorescent marker, CellMask $^{\mathrm{TM}}(R r=0.58)$ compared to full-length AC8 $(R r=0.77$; Fig. $5 \mathrm{c}, \mathrm{d})$. These results imply that glycosylation plays a partial role in trafficking AC8 to the plasma membrane. Time-lapse confocal imaging revealed that transiently transfected full-length AC8 distributed at the plasma membrane within approximately 48 -h post-transfection; an event which paralleled its N-linked glycosylation (Fig. 3Sa, b). These data suggest that N-linked glycosylation and AC8 confinement at the plasma membrane are likely to be interconnected events. c Single-cell Epac2-camps detection of cAMP in AC8 and AC8 $\Delta$ cav cells following SOCE. Maximum saturation (Max) was attained by addition of a cocktail of Forskolin $(10 \mu \mathrm{M})$ and IBMX $(100 \mu \mathrm{M})$ at $360 \mathrm{sec}$ (indicated by arrows). d Stimulation by SOCE (relative to Max). Results are plotted as \% of Max. Data are presented as mean + SEM

Transiently transfected AC $8 \Delta \mathrm{N}$-gly was localized outside lipid rafts (Pagano et al. 2009). However, the residence of AC8 in lipid rafts is necessary for its regulation by SOCE (Smith et al. 2002). Consequently, AC $8 \Delta \mathrm{N}$-gly was stably expressed and fractionated on sucrose gradients. Lipid raft isolation demonstrated that, unlike transiently transfected AC $8 \Delta \mathrm{N}$-gly, stably expressed AC $8 \Delta \mathrm{N}$-gly co-fractionated with caveolin1 and actin on sucrose gradients (Fig. 6a, b). The relative distribution of GFP-AC $8 \Delta \mathrm{N}$-gly and the actin cytoskeleton was then analysed by confocal imaging. GFPAC $8 \Delta \mathrm{N}$-gly showed considerable overlay with the actin signal $(R r=0.67)$, albeit to a lesser extent than full-length $\mathrm{AC} 8$, which indicates the existence of a significantly larger intracellular pool due to compromised targeting. Treatment with Lat B internalized AC8 $\Delta \mathrm{N}$-gly, which still maintained a degree of association with the actin signal $(R r=0.40)$. Furthermore, under these conditions, $\mathrm{AC} 8 \Delta \mathrm{N}$-gly aggregated and colocalized with caveolin 1 , mirroring the behaviour of AC8 $(R r=0.55$; Fig. $6 \mathrm{c}, \mathrm{d})$.

To further verify the association of AC $8 \Delta \mathrm{N}$-gly with lipid rafts, cholesterol was extracted with $\mathrm{M} \beta \mathrm{CD}$, a water-soluble 
$\mathbf{a}$
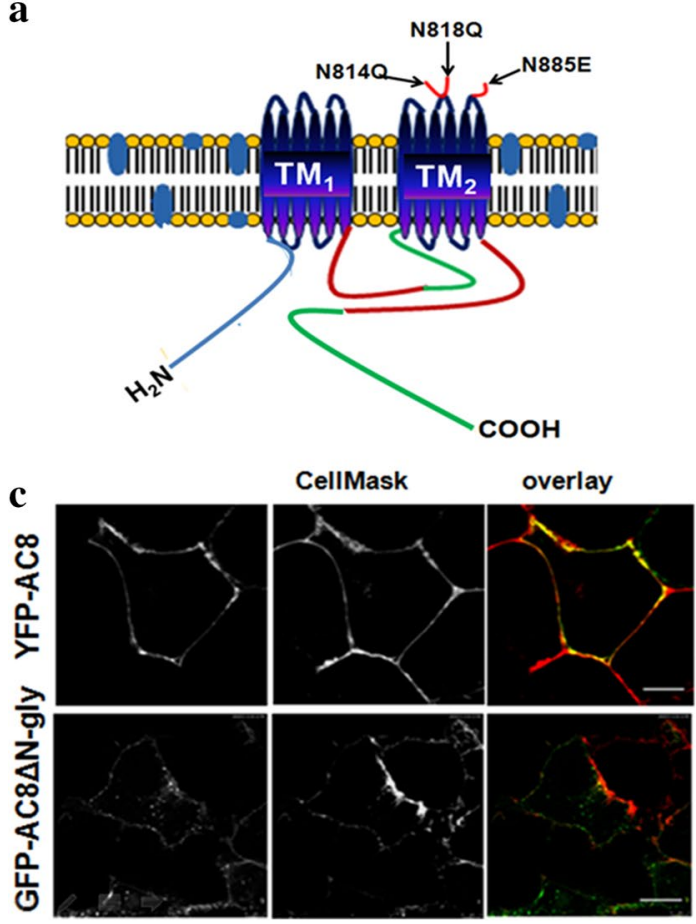

b
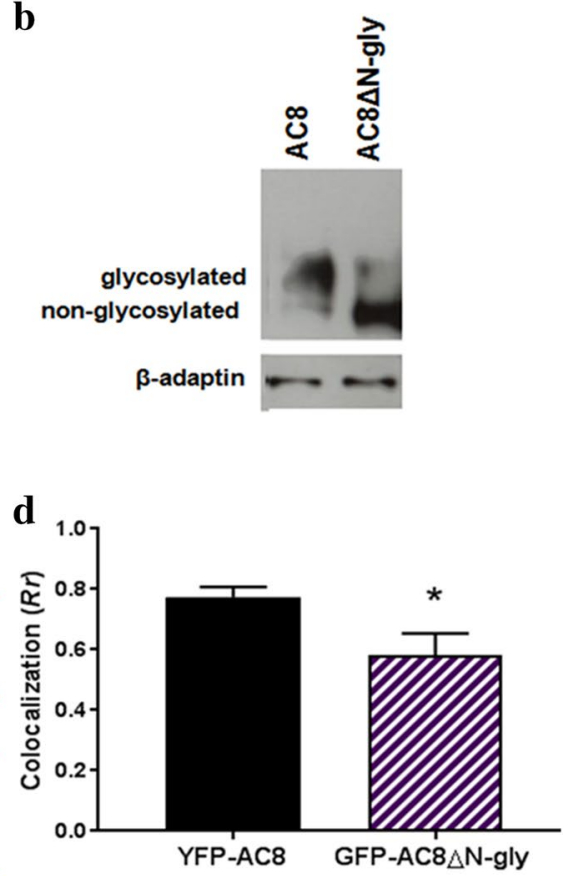

Fig. 5 N-linked glycosylation directs AC8 to the plasma membrane a Schematic representation of AC8 depicting its predicted topology. $\mathrm{AC} 8-\mathrm{Nt}$ is shown in blue, $\mathrm{C} 1 \mathrm{a}$ and $\mathrm{C} 2 \mathrm{a}$ domains are in red, $\mathrm{C} 1 \mathrm{~b}$ and $\mathrm{C} 2 \mathrm{~b}$ fragments are shown in green. The three asparagine residues that constitute potential $\mathrm{N}$-linked glycosylation sites located on the 5th and 6th extracellular loops within the second TM casette (shown by black arrows) were mutated to glutamine (N814 and N818) and glutamic acid (N885) to generate AC8 $\Delta \mathrm{N}$-gly. b Western blot analysis of crude membranes from HEK293 cells stably expressing AC8 and $\mathrm{AC} 8 \Delta \mathrm{N}$-gly (6\% acrylamide gels) probed for AC8 and $\beta$-adaptin by immunoblotting. c Confocal imaging of AC8 and AC8 $\Delta \mathrm{N}$-gly in overlay with CellMask ${ }^{\mathrm{TM}}$ Deep Red $(n=25-30$; scale bars represent $10 \mu \mathrm{m}$; optical section thickness $=0.773 \mu \mathrm{m})$. d Colocalization analysis $(R r)$ of $\mathbf{c}$. Data are presented as mean \pm SEM. (Color figure online) cyclic oligosaccharide which encloses a hydrophobic core acting as a carrier for amphipathic molecules such as cholesterol. Due to its cholesterol-binding properties, M $\beta C D$ has been broadly used to deplete cells of cholesterol (Zidovetzki and Levitan 2007). Treatment with M $\beta C D$ resulted in extrusion of AC $8 \Delta \mathrm{N}$-gly and caveolin 1 and an increase in their colocalization coefficient $(R r=0.62$; Fig. $4 \mathrm{Sa})$. These data indicate that GFP- AC $8 \Delta \mathrm{N}$-gly largely resides in lipid rafts.

Earlier single time-point measurements of cAMP in populations of cells suggested that $\mathrm{AC} 8 \Delta \mathrm{N}$-gly and AC8 display similar activation profiles. Slight, statistically insignificant differences in their responsiveness were attributed to dissimilarities in their levels of expression (Pagano et al. 2009). Given the inherent limitations in the spatio-temporal resolution of such measurements in populations of cells, we measured cAMP in single cells as a function of time using Epac2-camps. These real-time measurements revealed that AC $8 \Delta \mathrm{N}$-gly was less responsive statistically to SOCE than AC8 (Fig. 4Sb, c).

Summarized, these data point towards a role of N-glycosylation in appropriate AC8 distribution at the plasma membrane, which is of course a pre-requisite for its functionality. Thus, residence in lipid rafts although essential, does not entirely account for AC8 responsiveness to SOCE. Other factors such as plasma membrane targeting may contribute to AC functionality.

\section{AC8 is Glycosylated with Hybrid or Complex Glycans and Acquires Detergent Insolubility as It Travels Through Golgi}

Lipid rafts are proposed to form during transition via the Golgi through the association of cholesterol with sphingolipids (Brown and Rose 1992; Simons and Toomre 2000). In order to determine whether AC8 acquires detergent insolubility upon transiting through the Golgi we used BFA, a fungal metabolite which blocks the anterograde ER to Golgi transport and the secretory flux inside the Golgi (Donaldson et al. 1992; Klausner et al. 1992).

Following treatment with BFA and extraction with Triton $\mathrm{X}-100$, a pool of AC8 and caveolin1 shifted from the Triton $\mathrm{X}$ insoluble fractions to the soluble fractions on sucrose gradients (Fig. 7a, b). Solubilization of AC8 and caveolin1 may be explained by their entrapment in the ER due to blockage of ER to Golgi transport by BFA. Remarkably, treatment with BFA caused a pool of actin to dissociate from the 
$\mathbf{a}$
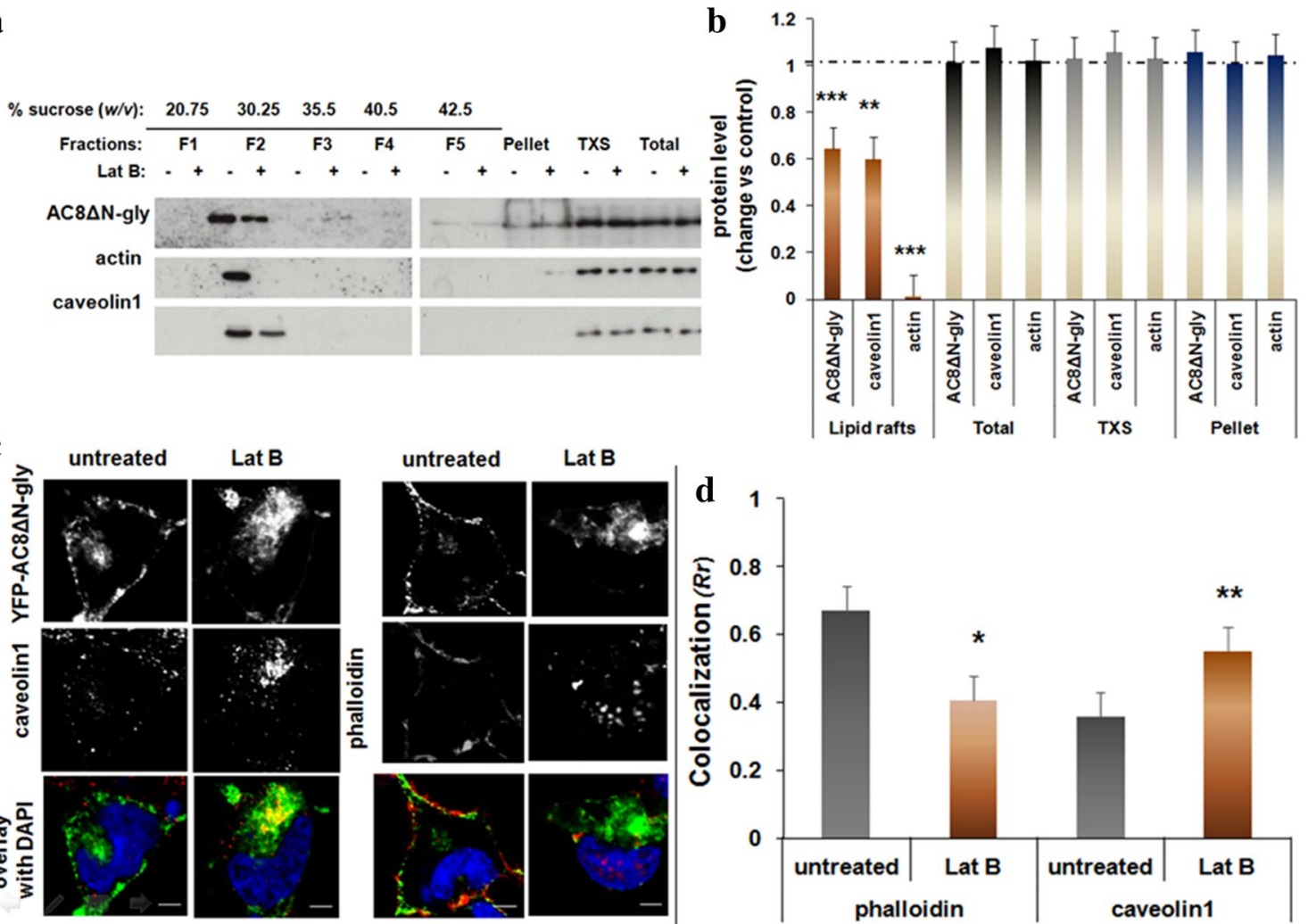

Fig. 6 AC8 $\Delta$ N-gly enriches in lipid rafts a HEK293 cells stably expressing GFP-AC $8 \Delta \mathrm{N}$-gly were homogenized in lysis buffer with $1 \%$ Triton X-100. The Triton X-100 soluble (TXS) and insoluble fractions were isolated, and $20 \mu \mathrm{g}$ of the extracts was analysed by SDS-PAGE. b Densitometric analysis (AU) of blots expressed as the ratio of protein after Lat $\mathrm{B}\left(2 \mu \mathrm{M}, 1 \mathrm{~h}, 37^{\circ} \mathrm{C}\right)$ treatment over con-

buoyant fractions (Fig. 7a, b). At the same time, the AC8 fluorescent signal re-located from the plasma membrane towards the cell interior, as determined by confocal imaging. At rest, the microfilaments appeared condensed at the plasma membrane; however, chronic treatment with BFA caused the cytoskeleton to collapse and aggregate towards the cell cytosol, adopting a more widespread appearance, perhaps due to de-polymerization (Fig. 7c). AC8 and actin signals, although partly overlapping, showed less colocalization than in resting cells $(R r=0.39$; Fig. $7 \mathrm{~d})$.

In order to better visualize the relative distribution of AC8 following treatment with BFA, cells were stained with wheat germ agglutinin (WGA). This lectin specifically binds sialic acid and $N$-acetyl-D-glucosamine contained in glycoproteins and glycolipids, making it an excellent option for staining plasma membrane and the Golgi (Chazotte 2011). In resting cells, AC8 showed a high degree of colocalization with the WGA signal at the plasma membrane $(R r=0.76$; Fig. 7c, d). Chronic treatment with BFA caused the WGA signal to relocate from the plasma membrane and the Golgi into a widespread structure, the ER (Fig. 7c). In parallel,

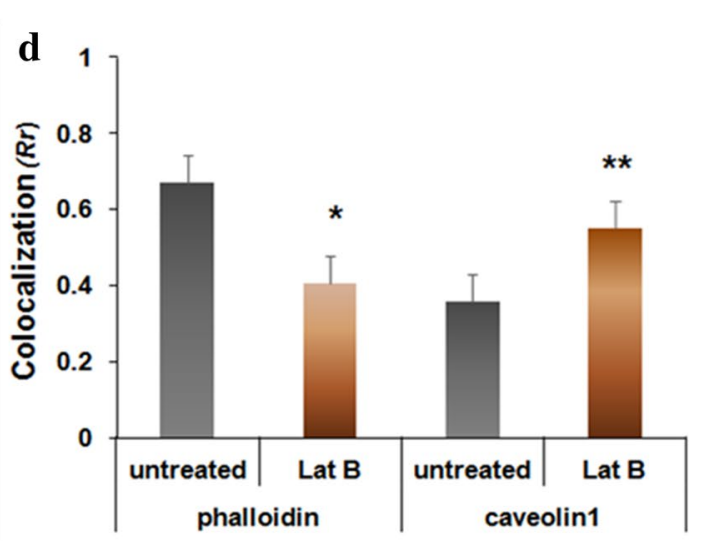

trol (DMSO). Data are presented as mean \pm SE $(n=3)$. c Confocal imaging of HEK293 cells expressing GFP-AC $8 \Delta \mathrm{N}$-gly treated or not with Lat B and stained with phalloidin $(n=10-13)$ or caveolin1 antibody $(n=12-15$; scale bars represent $10 \mu \mathrm{m}$; optical section thickness $=1.073 \mu \mathrm{m})$. d Colocalization analysis $(R r)$ of $\mathbf{c}$. Data are presented as mean \pm SEM

AC8 also redistributed from the plasma membrane displaying partial overlay with the WGA signal $(R r=0.55$; Fig. $7 \mathrm{~d})$.

A time course of BFA treatment revealed that the drug impaired AC8 glycosylation to some extent but did not affect its dimerization (Fig. 5Sa, b). Typically, glycoproteins are modified with high mannose $\mathrm{N}$-glycans co-translationally in the ER and they may be further modified with hybrid and complex N-glycans in the cis- and medial compartments of the Golgi (Vagin et al. 2009). Since BFA inhibits the ER to Golgi trafficking as well as the AC8 glycosylation pattern, our data suggest that AC8 is processed with hybrid and/or complex glycans. Thus, blockage of ER to Golgi trafficking impairs AC8 glycosylation, the assembly of AC8 microdomain and the integrity of the cytoskeleton.

\section{Discussion}

The essential residence of ACs at the plasma membrane is a neglected topic. Obviously for ACs to be regulated by G protein coupled receptor-based mechanisms or even by the 
$\mathbf{a}$

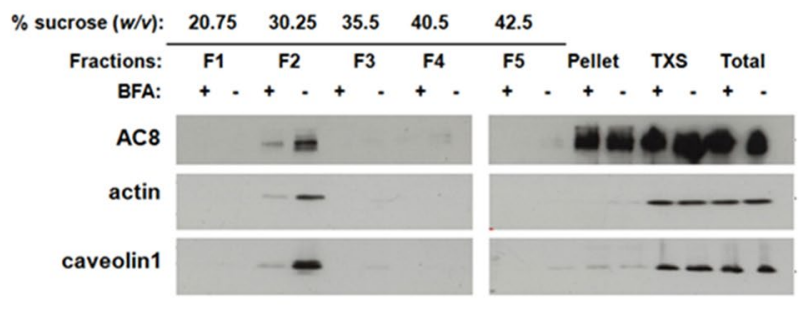

c

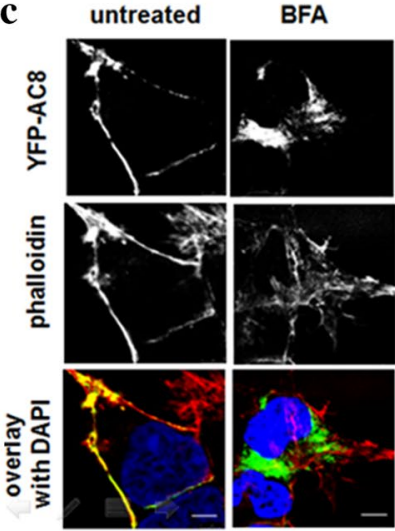

b
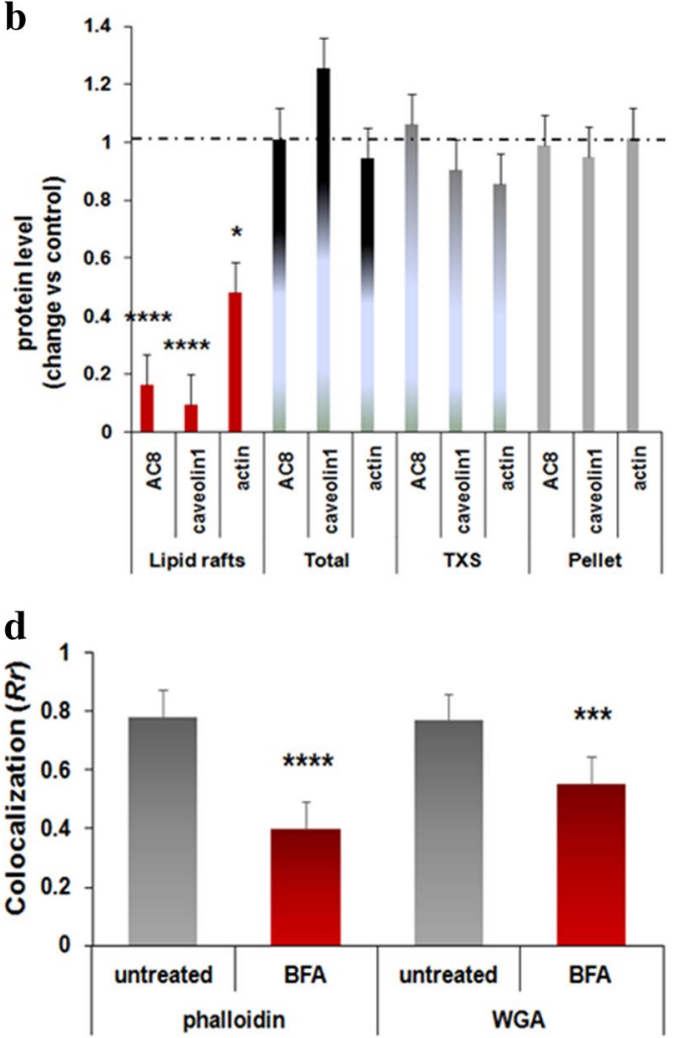

Fig. 7 Blockage of ER to Golgi trafficking decreases the association of AC8 with lipid rafts a HEK293 cells stably expressing YFP-AC8 were homogenized in lysis buffer with $1 \%$ Triton X-100. The Triton X-100 soluble (TXS) and insoluble fractions were isolated, and $20 \mu \mathrm{g}$ of the extracts was analysed by SDS-PAGE. b Densitometric analysis (AU) of blots expressed as the ratio of protein after BFA

elements of SOCE, the AC must encounter these regulators at the plasma membrane. However, what is not known and what is definitely understudied is how AC dwells at the plasma membrane and the influences that promote its proper targeting to the points where it will be regulated. In this study, we have used a number of cellular approaches to attempt to both consolidate and expand upon the various tacks that have emerged from previous studies of ACs or of other surface plasma membrane proteins. In this regard, we have considered that a dynamic interplay might pertain between a range of contributory elements. For instance, we know that the AC explored in the study, AC8, must necessarily reside in lipid rafts for its regulation by SOCE (Smith et al. 2002). Cholesterol-binding domains are sometimes a means for prolonging the associations of such raft targeted proteins (Lorent and Levental 2015). We also know that the AC, like many plasma membrane proteins, must be properly $\mathrm{N}$-glycosylated to be targeted to the plasma membrane (Gu et al. 2001; Vagin et al. 2009). Additionally, AC8 is found in association with caveolin 1 (Figs. 1, 2). Again, we
(5 $\mu \mathrm{g} / \mathrm{ml}$ ) treatment over control (DMSO). Data are presented as mean \pm SE $(n=3)$. c Confocal imaging of HEK293 cells expressing YFP-AC8 treated or not with BFA $(5 \mu \mathrm{g} / \mathrm{ml})$ and stained with phalloidin or WGA (scale bars represent $10 \mu \mathrm{m}$; optical section thickness $=1.073 \mu \mathrm{m})$. $\mathbf{d}$ Colocalization coefficient $(R r)$ of $\mathbf{c}$. Data are presented as mean \pm SEM

also know from earlier studies that AC8 undergoes essential associations with the actin cytoskeleton (Ayling et al. 2012). Consequently, here we have taken a comprehensive, dynamic approach where we try to consolidate these various influences into a comprehensive integrated sense of the trafficking and delivery of the AC to and from the plasma membrane.

We have made a number of observations of multiple factors contributing to the proper targeting of the AC, which when organized contextually allow a picture to emerge of a trafficking process that can be interrogated at various points and which allows a clear sense of flow to emerge. Very simply, AC8 binds both actin and caveolin1. At the same time, the cytoskeleton and caveolin 1 are critical determinants of the integrity of lipid rafts (Cohen et al. 2004; Head et al. 2014). From knock-down of caveolin 1 and disruption of the cytoskeleton with Lat B, both caveolin 1 and the cytoskeleton are seen to be crucial for maintaining the integrity of the AC8 microdomain (Figs. 1, 2). Additionally, the integrity of lipid rafts is required for proper AC8 functionality. 
Interestingly, dissociation of AC8 from lipid rafts following caveolin1 knock-down hyperactivates the enzyme (Fig. 1Sb, c). This result is consistent with previous findings assigning caveolins a role in tonic inhibition of AC (Toya et al. 1998; Allen et al. 2009).

During secretion from the Golgi, AC8, like many plasma membrane-targeted proteins (Vagin et al. 2009), is essentially $\mathrm{N}$-glycosylated with hybrid and/or complex glycans (Fig. 7, 5Sa, b); the pattern of glycosylation dictates its association with the plasma membrane and its functionality (Fig. 5, 4Sb, c). Interestingly, residence of AC8 in lipid rafts does not seem to depend on its glycosylation status: both $\mathrm{AC} 8 \Delta \mathrm{cav}$ and $\mathrm{AC} 8 \Delta \mathrm{N}$-gly remained associated with lipid rafts despite obvious defects in glycosylation and responsiveness (Figs. 4, 6). Critically, AC8 becomes an integral part of lipid rafts prior to its arrival to the plasma membrane (Fig. 7).

AC8 displays caveolin-binding motifs (CBM), which is a common means by which many raft-resident proteins are believed to be targeted to lipid rafts (Byrne et al. 2012). Mutation of these CBM disrupts proper post-translational processing (N-linked glycosylation) of AC8 (Fig. 3) and consequently negatively affects both its targeting to the plasma membrane and its responsiveness (Fig. 4c, d, 2Sa, b).

Our imaging experiments support colocalization of AC8 with caveolin 1-the best documented and widely accepted bona fide raft proxy, which was used to assess the quality of our biochemical preparation. Data collected following knock-down of caveolin1 represents further indirect evidence for AC8-caveolin1 association. These data alongside the identification of caveolin1-binding motifs in the primary structure of AC8 and the link of their mutation to AC8 processing and trafficking represent a strong body of evidence for the presence of AC8 in lipid rafts. Thus, in summary, the data gathered by our biochemical approach are supported by high-resolution confocal microscopy, mutagenesis, pharmacological tools and FRET experiments as well as the responsiveness of AC8 to physiological stimuli in addition to previously published data.

Based on these explorations, we envisage a model for AC8 processing, trafficking, assembly into cholesterolrich domains and targeting to the plasma membrane lipid rafts. We conclude that $\mathrm{AC} 8$ traffics to and from the plasma membrane in a cyclical process that involves synthesis in the ER, progression through the Golgi where it is further N-glycosylated. Somewhere along the secretory pathway, AC8 becomes associated with caveolin1 and is targeted to internal rafts which themselves traffic to and from the plasma membrane (Simons and Ikonen 1997; Cooper and Tabbasum 2014). In addition, caveolin 1 may control AC8 functionality at the plasma membrane either directly or indirectly via interactions with AC8. Thus, caveolin 1 exerts complex effects on AC8 processing, trafficking and responsiveness.
This model, within the context of trafficking and processing of membrane proteins, offers an efficient explanation for the complex assembly and organization of AC8 in lipid rafts.

The realization of the range of the potential AC-caveolin interactions raises further questions. Resolving the details of such interactions may help to better understand the dynamics of AC movement and its integration in lipid rafts. From the current data, we cannot conclude whether caveolin1 and AC 8 bind via a direct or an indirect interaction. Hence, future studies should be directed towards understanding the specific mechanisms underlying these interactions and their contribution to the secretion of ACs, their targeting and functionality in lipid rafts.

Acknowledgements This research was supported in part by the Wellcome Trust (RG31760), the Newton Trust University of Cambridge and the Gates Cambridge Trust.

\section{Compliance with Ethical Standards}

Conflict of interest The authors declare that they have no conflict of interest. This research was supported in part by the Wellcome Trust (RG31760), the Newton Trust University of Cambridge and the Gates Cambridge Trust.

Open Access This article is distributed under the terms of the Creative Commons Attribution 4.0 International License (http://creativeco mmons.org/licenses/by/4.0/), which permits unrestricted use, distribution, and reproduction in any medium, provided you give appropriate credit to the original author(s) and the source, provide a link to the Creative Commons license, and indicate if changes were made.

\section{References}

Aaron JS, Taylor AB, Chew TL (2018) Image co-localization-cooccurrence versus correlation. J Cell Sci. https://doi.org/10.1242/ jcs. 211847

Adler J, Parmryd I (2010) Quantifying colocalization by correlation: the Pearson correlation coefficient is superior to the Mander's overlap coefficient. Cytometry 77(8):733-742

Allen JA, Yu JZ, Dave RH, Bhatnagar A, Roth BL, Rasenick MM (2009) Caveolin-1 and lipid microdomains regulate Gs trafficking and attenuate Gs/adenylyl cyclase signaling. Mol Pharmacol 76(5):1082-1093

Ayling LJ, Briddon SJ, Halls ML, Hammond GR, Vaca L, Pacheco J, Hill SJ, Cooper DMF (2012) Adenylyl cyclase AC8 directly controls its micro-environment by recruiting the actin cytoskeleton in a cholesterol-rich milieu. J Cell Sci 125(4):869-886

Bastiani M, Parton RG (2010) Caveolae at a glance. J Cell Sci 123(22):3831-3836

Bonifacino JS, Glick BS (2004) The mechanisms of vesicle budding and fission. Cell 116(2):153-166

Brown DA, London E (1998) Functions of lipid rafts in biological membranes. Annu Rev Cell Dev Biol 14:111-136

Brown DA, Rose JK (1992) Sorting of GPI-anchored proteins to glycolipid-enriched membrane subdomains during transport to the apical cell surface. Cell 68(3):533-544 
Byrne DP, Dart C, Rigden DJ (2012) Evaluating caveolin interactions: do proteins interact with the caveolin scaffolding domain through a widespread aromatic residue-rich motif? PLoS ONE 7(9):e44879

Cali JJ, Zwaagstra JC, Mons N, Cooper DMF, Krupinski J (1994) Type VIII adenylyl cyclase. A $\mathrm{Ca}^{2+} /$ calmodulin-stimulated enzyme expressed in discrete regions of rat brain. J Biol Chem 269(16):12190-12195

Chazotte B (2011) Labeling membrane glycoproteins or glycolipids with fluorescent wheat germ agglutinin. Cold Spring Harb Protoc. https://doi.org/10.1101/pdb.prot5623

Cohen AW, Hnasko R, Schubert W, Lisanti MP (2004) Role of caveolae and caveolins in health and disease. Physiol Rev 84(4):1341-1379

Conti AC, Maas JW Jr, Muglia LM, Dave BA, Vogt SK, Tran TT, Rayhel EJ, Muglia LJ (2007) Distinct regional and subcellular localization of adenylyl cyclases type 1 and 8 in mouse brain. Neuroscience 146(2):713-729

Cooper DMF (2003) Regulation and organization of adenylyl cyclases and cAMP. Biochem J 375(3):517-529

Cooper DMF, Crossthwaite AJ (2006) Higher-order organization and regulation of adenylyl cyclases. Trends Pharmacol Sci 27(8):426-431

Cooper DMF, Tabbasum VG (2014) Adenylate cyclase-centred microdomains. Biochem J 462(2):199-213

Couet J, Li S, Okamoto T, Ikezu T, Lisanti MP (1997) Identification of peptide and protein ligands for the caveolin-scaffolding domain. Implications for the interaction of caveolin with caveolae-associated proteins. J Biol Chem 272(10):6525-6533

Crossthwaite AJ, Seebacher T, Masada N, Ciruela A, Dufraux K, Schultz JE, Cooper DMF (2005) The cytosolic domains of $\mathrm{Ca}^{2+}$-sensitive adenylyl cyclases dictate their targeting to plasma membrane lipid rafts. J Biol Chem 280(8):6380-6391

Delint-Ramirez I, Willoughby D, Hammond GR, Ayling LJ, Cooper DMF (2015) Palmitoylation targets AKAP79 protein to lipid rafts and promotes its regulation of calcium-sensitive adenylyl cyclase type 8. J Biol Chem 286(38):32962-32975

Doherty GJ, McMahon HT (2009) Mechanisms of endocytosis. Annu Rev Biochem 78:857-902

Donaldson JG, Finazzi D, Klausner RD (1992) Brefeldin A inhibits Golgi membrane-catalysed exchange of guanine nucleotide onto ARF protein. Nature 360(6402):350-352

Dupree P, Parton RG, Raposo G, Kurzchalia TV, Simons K (1993) Caveolae and sorting in the trans-Golgi network of epithelial cells. EMBO J 12(4):1597-1605

Fagan KA, Mahey R, Cooper DMF (1996) Functional co-localization of transfected $\mathrm{Ca}^{2+}$-stimulable adenylyl cyclases with capacitative $\mathrm{Ca}^{2+}$ entry sites. J Biol Chem 271(21):12438-12444

Feige MJ, Hendershot LM (2013) Quality control of integral membrane proteins by assembly-dependent membrane integration. Mol Cell 51(3):297-309

Ferguson GD, Storm DR (2004) Why calcium-stimulated adenylyl cyclases? Physiology (Bethesda) 19:271-276

Gagescu R, Demaurex N, Parton RG, Hunziker W, Huber LA, Gruenberg J (2000) The recycling endosome of Madin-Darby canine kidney cells is a mildly acidic compartment rich in raft components. Mol Biol Cell 11(8):2775-2791

Gu C, Cali JJ, Cooper DMF (2002) Dimerization of mammalian adenylate cyclases. Eur J Biochem 269(2):413-421

Gu C, Sorkin A, Cooper DMF (2001) Persistent interactions between the two transmembrane clusters dictate the targeting and functional assembly of adenylyl cyclase. Curr Biol 11(3):185-190

Head BP, Patel HH, Insel PA (2014) Interaction of membrane/lipid rafts with the cytoskeleton: impact on signaling and function: membrane/lipid rafts, mediators of cytoskeletal arrangement and cell signaling. Biochim Biophys Acta 1838(2):532-545
Johnstone TB, Agarwal SR, Harvey RD, Ostrom RS (2018) cAMP signaling compartmentation: adenylyl cyclases as anchors of dynamic signaling complexes. Mol Pharmacol 93(4):270-276

Klausner RD, Donaldson JG, Lippincott-Schwartz J (1992) Brefeldin A: insights into the control of membrane traffic and organelle structure. J Cell Biol 116(5):1071-1080

Kurzchalia TV, Dupree P, Parton RG, Kellner R, Virta H, Lehnert M, Simons K (1992) VIP21, a 21-kD membrane protein is an integral component of trans-Golgi-network-derived transport vesicles. J Cell Biol 118(5):1003-1014

Lagerholm BC, Weinreb GE, Jacobson K, Thompson NL (2005) Detecting microdomains in intact cell membranes. Annu Rev Phys Chem 56:309-336

Levental I, Veatch S (2016) The continuing mystery of lipid rafts. J Mol Biol 428(24):4749-4764

Lorent JH, Levental I (2015) Structural determinants of protein partitioning into ordered membrane domains and lipid rafts. Chem Phys Lipids 192:23-32

Mohamed A, Shah A, Chen D, Hill MM (2018) RaftProt V2: understanding membrane microdomain function through lipid raft proteomes. Nucleic Acids Res 47:D459-D463

Muriel O, Echarri A, Hellriegel C, Pavón DM, Beccari L, Del Pozo MA (2011) Phosphorylated filamin A regulates actin-linked caveolae dynamics. J Cell Sci 124(16):2763-2776

Nikolaev VO, Bunemann M, Hein L, Hannawacker A, Lohse MJ (2004) Novel single chain cAMP sensors for receptor-induced signal propagation. J Biol Chem 279(36):37215-37218

Pagano M, Clynes MA, Masada N, Ciruela A, Ayling LJ, Wachten S, Cooper DMF (2009) Insights into the residence in lipid rafts of adenylyl cyclase AC8 and its regulation by capacitative calcium entry. Am J Physiol Cell Physiol 296(3):C607-19

Parekh AB, Putney JW Jr (2005) Store-operated calcium channels. Physiol Rev 85(2):757-810

Parton RG, Howes MT (2010) Revisiting caveolin trafficking: the end of the caveosome. J Cell Biol 191(3):439-441

Pike JA, Styles IB, Rappoport JZ, Heath JK (2017) Quantifying receptor trafficking and colocalization with confocal microscopy. Methods 115:42-54

Pol A, Martin S, Fernández MA, Ingelmo-Torres M, Ferguson C, Enrich C, Parton RG (2005) Cholesterol and fatty acids regulate dynamic caveolin trafficking through the Golgi complex and between the cell surface and lipid bodies. Mol Biol Cell 16(4):2091-2105

Razani B, Woodman SE, Lisanti MP (2002) Caveolae: from cell biology to animal physiology. Pharmacol Rev 54(3):431-467

Sato M, Hutchinson DS, Halls ML, Furness SG, Bengtsson T, Evans BA, Summers RJ (2012) Interaction with caveolin-1 modulates $\mathrm{G}$ protein coupling of mouse $\beta 3$-adrenoceptor. J Biol Chem 287(24):20674-20688

Sezgin E, Levental I, Mayor S, Eggeling C (2017) The mystery of membrane organization: composition, regulation and roles of lipid rafts. Nat Rev Mol Cell Biol 18(6):361-374

Shah A, Chen D, Boda AR, Foster LJ, Davis MJ, Hill MM (2015) RaftProt: mammalian lipid raft proteome database. Nucleic Acids Res 43:D335-D338

Simons K, Ikonen E (1997) Functional rafts in cell membranes. Nature 387(6633):569-572

Simons K, Toomre D (2000) Lipid rafts and signal transduction. Nat Rev Mol Cell Biol 1(1):31-39

Simons K, Vaz WL (2004) Model systems, lipid rafts, and cell membranes. Annu Rev Biophys Biomol Struct 33:269-295

Smith KE, Gu C, Fagan KA, Hu B, Cooper DMF (2002) Residence of adenylyl cyclase type 8 in caveolae is necessary but not sufficient for regulation by capacitative $\mathrm{Ca}^{2+}$ entry. $\mathrm{J}$ Biol Chem 277(8):6025-6031 
Spector I, Shochet NR, Blasberger D, Kashman Y (1989) Latrunculinsnovel marine macrolides that disrupt microfilament organization and affect cell growth: I. Comparison with cytochalasin D. Cell Motil Cytoskeleton 13(3):127-144

Swaney JS, Patel HH, Yokoyama U, Head BP, Roth DM, Insel PA (2006) Focal adhesions in (myo)fibroblasts scaffold adenylyl cyclase with phosphorylated caveolin. J Biol Chem 281(25): 17173-17179

Toya Y, Schwencke C, Couet J, Lisanti MP, Ishikawa Y (1998) Inhibition of adenylyl cyclase by caveolin peptides. Endocrinology 139(4):2025-2031

Vagin O, Kraut JA, Sachs G (2009) Role of N-glycosylation in trafficking of apical membrane proteins in epithelia. Am J Physiol Renal Physiol 296(3):F459-F469

Willoughby D, Masada N, Wachten S, Pagano M, Halls ML, Everett KL, Ciruela A, Cooper DMF (2010) A-kinase anchoring protein 79/150 interacts with adenylyl cyclase type 8 and regulates $\mathrm{Ca}^{2+}$-dependent cAMP synthesis in pancreatic and neuronal systems. J Biol Chem 285:20328-20342
Willoughby D, Everett KL, Halls ML, Pacheco J, Skroblin P, Vaca L, Klussmann E, Cooper DMF (2012) Direct binding between Orai1 and AC8 mediates dynamic interplay between $\mathrm{Ca}^{2+}$ and cAMP signaling. Sci Signal 5(219):ra29

Yarmola EG, Somasundaram T, Boring TA, Spector I, Bubb MR (2000) Actin-Latrunculin A structure and function. Differential modulation of actin-binding protein function by latrunculin A. J Biol Chem 275(36):28120-28127

Zidovetzki R, Levitan I (2007) Use of cyclodextrins to manipulate plasma membrane cholesterol content: evidence, misconceptions and control strategies. Biochim Biophys Acta 1768(6):1311-1324

Publisher's Note Springer Nature remains neutral with regard to jurisdictional claims in published maps and institutional affiliations. 